\title{
Avalanche dynamics in higher-dimensional fiber bundle models
}

\author{
Zsuzsa Danku, ${ }^{1}$ Géza Ódor, ${ }^{2}$ and Ferenc Kun ${ }^{1, *}$ \\ ${ }^{1}$ Department of Theoretical Physics, University of Debrecen, P.O. Box 5, H-4010 Debrecen, Hungary \\ ${ }^{2}$ MTA-MFA-EK, Center for Energy Research of the Hungarian Academy of Sciences, P.O. Box 49, H-1121 Budapest, Hungary
}

(Received 22 May 2018; published 15 October 2018)

\begin{abstract}
We investigate how the dimensionality of the embedding space affects the microscopic crackling dynamics and the macroscopic response of heterogeneous materials. Using a fiber bundle model with localized load sharing, computer simulations are performed from one to eight dimensions slowly increasing the external load up to failure. Analyzing the constitutive curve, fracture strength, and avalanche statistics of bundles we demonstrate that a gradual crossover emerges from the universality class of localized behavior to the mean field class of fracture as the embedding dimension increases. The evolution between the two universality classes is described by an exponential functional form. Simulations revealed that the average temporal profile of crackling avalanches evolves with the dimensionality of the system from a strongly asymmetric shape to a symmetric parabola characteristic for localized stresses and homogeneous stress fields, respectively.
\end{abstract}

DOI: 10.1103/PhysRevE.98.042126

\section{INTRODUCTION}

A large variety of heterogeneous materials respond to a slow external driving in a jerky way where sudden outbreaks of activity are separated by silent periods [1,2]. From the propagation of imbibition fronts in heterogeneous materials [3], through dislocation bursts of plastically deforming crystals [4], and Barkhausen noise in ferromagnets [5], to fracture phenomena [6,7] and earthquakes [8], crackling noise has been observed over a broad range of length scales. It was found that crackling noise is characterized by scaling laws, i.e., the statistics of the quantities of single bursts is described by power-law distributions which may be the fingerprint of an underlying phase transition $[1,5,9]$.

Recently, it has been pointed out that the average temporal profile is a fundamental feature of crackling avalanches. Experimental and theoretical studies have revealed that the precise shape of the average profile of bursts is sensitive to the details of the physics of the system, and it encodes valuable information about the underlying intermittent dynamics of pulse generation [8,10-13]. For the fracture of heterogeneous materials careful experiments have been performed where the temporal evolution of individual bursts formed at a propagating crack front was determined by direct optical observation using high-speed imaging $[6,14,15]$. These investigations provided symmetric parabolic profiles mainly attributed to long-range elastic forces acting along the crack front [6]. Measurements of magnetic noise induced by the dynamic fracture of steal revealed similar pulse profiles, however, with a right-handed asymmetry $[7,16]$. The front propagation was modeled as the driven motion of an elastic line in a disordered environment of pinning centers. Varying the range of interaction it was found that the degree of asymmetry depends on the range of stress redistribution, i.e., profiles evolve with the universality class of fracture from a strongly asymmetric shape (localized

\footnotetext{
*ferenc.kun@science.unideb.hu
}

interaction) to a symmetric parabola (long-range interaction) [6]. Simulation studies of the dynamics of breaking bursts in the fiber bundle model underlined the general validity of this behavior [17].

In the present paper we take the opposite strategy and address the question how the dimensionality of the sample affects the fracture process when the range of interaction is kept constant. We performed computer simulations in the framework of a fiber bundle model with nearest-neighbor load sharing after local failure events varying the dimensionality of the system from one to eight. On both the macroand microscales the system exhibits a crossover between the universality classes of localized behavior and the mean field class of fracture phenomena. We show that this evolution is described by a genuine exponential form. The temporal profile of breaking avalanches can be well described by the scaling form suggested in Ref. [6] where the parameters clearly confirm the crossover between the two universality classes. Our study shows that the upper critical dimension of the fracture of heterogeneous materials is infinite in agreement with a recent theoretical prediction [18]. We give numerical evidence that the critical exponents change as an exponential function of the dimension.

\section{LOCAL LOAD-SHARING FIBER BUNDLE MODEL IN ONE TO EIGHT DIMENSIONS}

The fiber bundle model provides an efficient modeling framework for the fracture of heterogeneous materials [19-21]. In spite of its simplicity it captures the essential ingredients of fracture phenomena allowing also for analytical solutions for the most important quantities [22,23]. The classical fiber bundle model consists of $N$ parallel fibers which are organized on a regular lattice. In $D=1$ fibers are placed equidistantly next to each other along a line, while in $D=2$ the fibers are assigned to the sites of a square lattice of side length $L$. The fibers are assumed to have a perfectly brittle 
behavior, i.e., they exhibit a linearly elastic response with a Young modulus $E$ up to breaking at a threshold load $\sigma_{t h}$. The Young modulus is assumed to be constant $E=1$ such that the disorder of the material is solely represented by the randomness of the breaking threshold $\sigma_{t h}$ : to each fiber a threshold value is assigned $\sigma_{t h}^{i}, i=1, \ldots, N$ sampled from the probability density $p\left(\sigma_{t h}\right)$. In the present calculations we used exponentially distributed breaking thresholds

$$
p\left(\sigma_{t h}\right)=\frac{1}{\lambda} e^{-\sigma_{t h} / \lambda}
$$

over the range $0 \leqslant \sigma_{t h}<+\infty$. The scale parameter was fixed to $\lambda=2$ in all calculations.

In $D \leqslant 2$ the bundle is loaded in the direction parallel to the fibers, which represents the uniaxial loading of a barshaped specimen. Under stress-controlled loading, when the local load on a fiber reaches its failure strength the fiber breaks and its load has to be redistributed over the remaining intact fibers. We assume localized load sharing (LLS) so that the load of a broken fiber is redistributed equally over its intact nearest neighbors in the square lattice [24-26]. When the breaking fiber is entirely surrounded by intact ones in the square lattice, four fibers share the load; however, when the breaking fiber is at the perimeter of a growing broken cluster (crack) typically two or three fibers receive the excess of the load. As a consequence, high stress concentration builds up along the perimeter of cracks, and local stress fluctuations develop.

In higher dimensions $D>2$ the generalization of the model is straightforward, although it does not have a direct relevance for practical applications: the fibers are assigned to sites of cubic lattices, and the load on them is represented by a scalar variable. After failure events the load is redistributed over the intact nearest-neighboring sites along the edges of the lattice. The emerging stress concentration is controlled by the coordination number $z$ of the underlying lattice, which depends on the embedding dimension as $z=2 D$ in our setup. In all dimensions periodic boundary conditions are implemented in all lattice directions.

Computer simulations were performed by quasistatically increasing the external load $\sigma$, which is realized by increasing $\sigma$ to provoke the breaking of a single fiber. After the fiber has been removed its load gets redistributed according to the rules discussed above. The enhanced load on the neighboring fibers may induce further breaking, which is followed again by a local stress redistribution. As the result of this repeated breaking and stress redistribution the breaking of a single fiber can give rise to an avalanche of breakings. The loading process stops when a catastrophic avalanche is triggered in which all remaining intact fibers break.

In order to keep the problem numerically tractable for the dimensions $D=1,2,3,4,5,6,7,8$ the lattice size was set to $L=4084101,2021,159,45,21,13,9,7$, which ensures nearly the same number of fibers in all dimensions. To obtain reliable results statistical averaging was done over $K=5000$ simulations.

\section{MACROSCOPIC RESPONSE}

The mean field limit of FBMs is realized by the equal load sharing (ELS) of the load of broken fibers over the intact

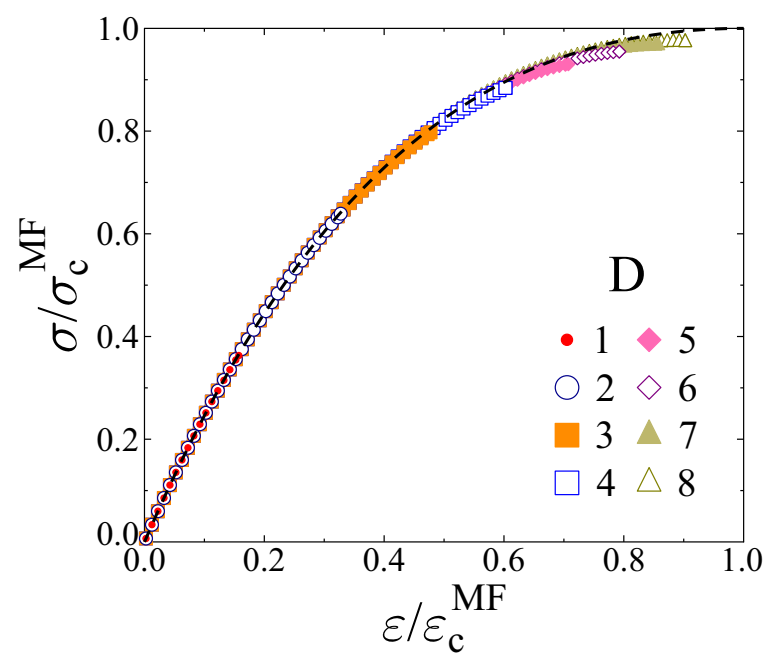

FIG. 1. The constitutive curve of the system for different dimensions $D$ scaled with the mean field critical strain $\varepsilon_{c}^{M F}$ and stress $\sigma_{c}^{M F}$. The mean field solution (2) is represented by the dashed line.

ones. Under ELS conditions all intact fibers receive the same amount of load irrespective of their distance from the broken one. It follows that no stress fluctuations can emerge, all fibers keep the same load during the entire loading process. Hence, in the mean field limit the random strength of fibers is the only source of disorder in the system. For ELS the macroscopic stress-strain relation $\sigma(\varepsilon)$ of the bundle can simply be obtained from the general expression $\sigma=E \varepsilon[1-P(E \varepsilon)]$. Here $P(x)$ denotes the cumulative distribution of the failure thresholds so that the term $1-P(E \varepsilon)$ yields the fraction of intact fibers which all keep the same load $E \varepsilon$. Substituting the exponential distribution (1) we obtain

$$
\sigma=E \varepsilon e^{-E \varepsilon / \lambda}
$$

In Fig. 1 the curve of Eq. (2) is presented up to the maximum where catastrophic failure occurs under stress controlled loading. The constitutive curve has a quadratic maximum the value $\sigma_{c}$ and position $\varepsilon_{c}$ of which determine the fracture stress and strain of the bundle, respectively:

$$
\begin{gathered}
\sigma_{c}^{M F}=\lambda / e, \\
\varepsilon_{c}^{M F}=\lambda / E .
\end{gathered}
$$

Note that the fracture strength $\sigma_{c}$ and $\varepsilon_{c}$ of FBMs depend on the system size $N$ even in the mean field limit [27-29]. Although the convergence is rapid, strictly speaking the above expressions give the bundle strength in the limit of infinite system size.

For finite dimensions $D$ the $\sigma(\varepsilon)$ curves were determined by computer simulations averaging over a large number of loading processes with different realizations of the threshold disorder. It can be seen in Fig. 1 that for all dimensions $D$ the mechanical response $\sigma(\varepsilon)$ of LLS bundles follows the mean field solution (2). However, for low-dimensional bundles the curves stop significantly earlier, implying a lower fracture strength and a higher degree of brittleness. As $D$ increases the LLS constitutive response completely recovers the mean 


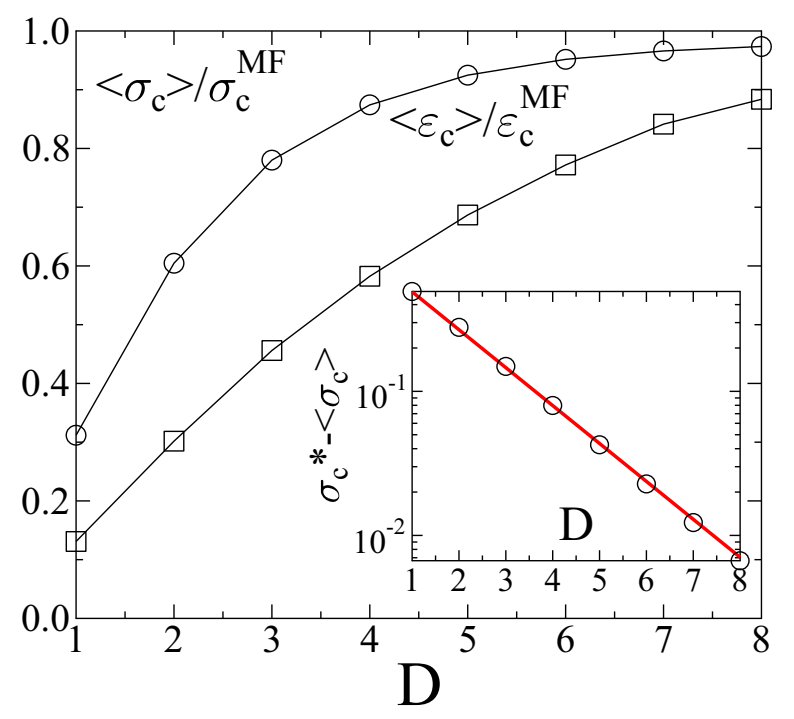

FIG. 2. The average fracture stress $\left\langle\sigma_{c}\right\rangle$ and strain $\left\langle\varepsilon_{c}\right\rangle$ obtained by directly averaging the stress and strain of the last stable configuration of the system in stress-controlled quasistatic loading simulations. The strength values are normalized by their mean field counterpart. Inset: subtracting a constant from the fracture stress straight line is obtained on a semilogarithmic plot.

field behavior. This tendency becomes more transparent when analyzing the average fracture stress $\left\langle\sigma_{c}\right\rangle$ and strain $\left\langle\varepsilon_{c}\right\rangle$ as a function of $D$. In Fig. 2 both quantities gradually converge to their mean field counterpart; however, the convergence is somewhat faster for $\left\langle\sigma_{c}\right\rangle$. For the highest dimension $D=8$ considered only a few percent difference is observed from the value of $\sigma_{c}^{M F}$ of Eq. (3).

The most remarkable result is that the convergence to the mean field limit can be described by an exponential form. The inset of Fig. 2 demonstrates that subtracting a constant $\sigma_{c}^{*}$ from $\left\langle\sigma_{c}\right\rangle$ a straight line is obtained on a semilogarithmic representation, which implies the functional form

$$
\left\langle\sigma_{c}\right\rangle(D)=\sigma_{c}^{*}+A \exp \left(-D / D^{*}\right) .
$$

Here $D^{*}$ denotes a characteristic value of the dimension. Formally, $\sigma_{c}^{*}$ is a free parameter in Eq. (5) which was tuned to $\sigma_{c}^{*}=0.725 \pm 0.015$ to obtain the best straight line in Fig. 2. Note that this value falls very close to $\sigma_{c}^{M F}=$ 0.735 . The characteristic dimension was obtained by fitting $D^{*}=1.65 \pm 0.06$.

The results show that in higher dimensions the role of stress fluctuations is diminishing in the fracture process and the behavior of the system gradually approaches the one of the completely homogeneous stress field of the infinite dimensional ELS solution. It follows that, in spite of the highly localized stress redistribution, in high $D$ the stochastic breaking process is completely controlled by the quenched disorder of the failure strength of fibers.

\section{AVALANCHE DYNAMICS}

The bundle is loaded in a quasistatic way such that the external load is increased to provoke solely the breaking of a single fiber. In the simulations the cascade of breaking fibers,

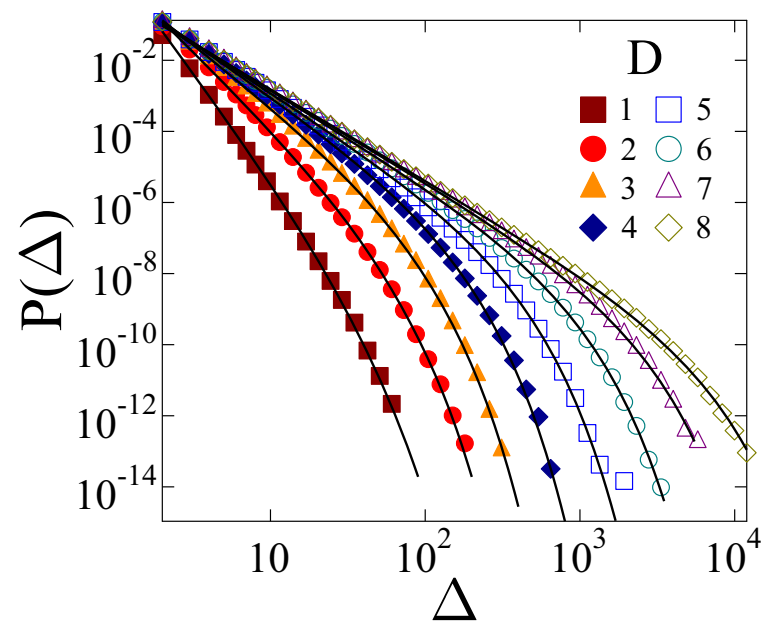

FIG. 3. The size distribution of bursts $p(\Delta)$ for all dimensions considered. The continuous lines represent best fits with the functional form (6).

emerging due to the repeated steps of load redistribution and breaking, is followed until it stops while keeping the external load fixed. The size $\Delta$ of avalanches is characterized by the number of fibers breaking in the avalanche. These breaking avalanches are analogous to crackling bursts measured in experiments with acoustic [30,31] or electromagnetic [7,16] techniques. Under simple geometrical conditions such as during the slow propagation of a planar crack direct optical observation has also proven very successful [6,32,33].

The probability distribution $p(\Delta)$ of avalanche sizes is presented in Fig. 3. In all dimensions $D$ the probability density $p(\Delta)$ is described by the same functional form, i.e., powerlaw distributions are obtained followed by an exponential cutoff

$$
p(\Delta) \sim \Delta^{-\tau} \exp \left(-\Delta / \Delta^{*}\right)
$$

where both the exponent $\tau$ and the characteristic burst size $\Delta^{*}$ depend on the dimensionality $D$ of the bundle. In Fig. 3, Eq. (6) provides excellent fits of the numerical data where the exponent $\tau$ decreases while $\Delta^{*}$ increases with $D$. The result implies that in higher dimensions the system can tolerate larger avalanches without catastrophic collapse. It has been shown by analytical calculations that in mean field FBMs the size distribution exponent takes the value $\tau_{M F}=5 / 2$, which has proven to be universal for a broad class of threshold distributions [20,22,34]. For our LLS system it can be observed in Fig. 4 that $\tau$ has high values in low dimensions, but with increasing $D$ it approaches the mean field exponent. The inset of Fig. 4 demonstrates that the convergence is again described by an exponential form similar to Eq. (5)

$$
\tau(D)=\tau^{*}+B \exp \left(-D / D^{*}\right)
$$

where best fit was obtained with the same value of the characteristic dimension $D^{*}=1.65 \pm 0.06$ as for the fracture strength (5). The value of $\tau^{*}$ providing the best straight line is $\tau^{*}=2.52 \pm 0.04$, which falls very close to the mean field exponent $\tau_{M F}$. 


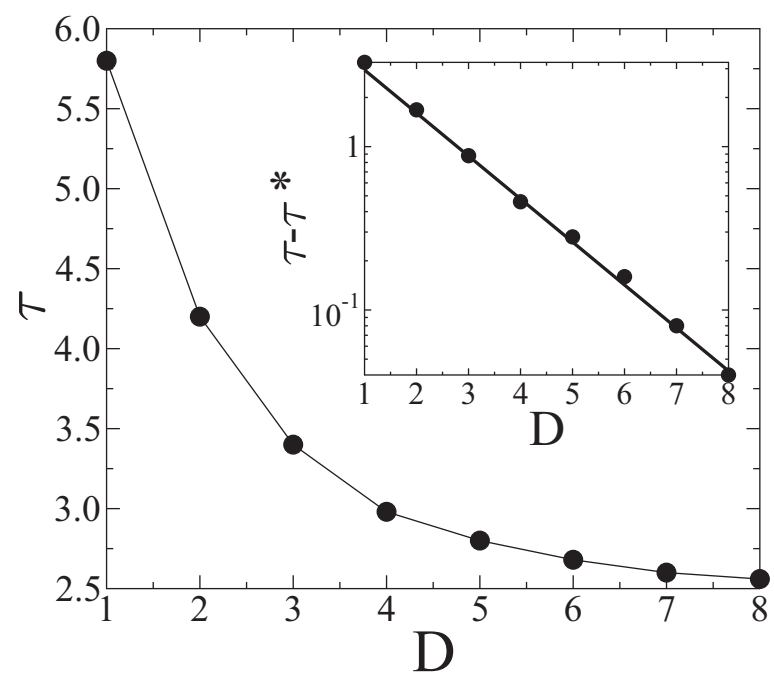

FIG. 4. The size distribution exponent $\tau$ of breaking avalanches obtained by fitting with the form (6). Inset: subtracting a proper value $\tau^{*}$ from $\tau$ a straight line is obtained on a semilogarithmic plot.

\section{TEMPORAL PROFILE OF AVALANCHES}

Recently we have shown that breaking avalanches in fiber bundles have a complex time evolution [17]: an avalanche typically starts with the breaking of a single fiber which in turn triggers the breaking of one or two additional fibers after load redistribution. The subsequent load redistribution steps involve a larger and larger number of fibers giving rise to a spatial spreading of the avalanche. The avalanche stops when all the fibers involved in the last redistribution step are able to sustain the elevated load. This dynamics implies that avalanches are composed of discrete growth steps of size $\Delta_{s}$, which is the number of fibers breaking in a single load redistribution step. The total number of subsequent redistribution-breaking steps defines the duration $W$ of the avalanche. The time evolution of a single burst of size $\Delta=8705$ and duration $W=264$ is illustrated in Fig. 5 for a three-dimensional bundle, where cubes represent fibers. The color code corresponding to the growth steps of the avalanche facilitates following the breaking sequence. The temporal evolution of an avalanche is characterized by the $\Delta_{s}(u)$ function, where $u$ is a time variable taking integer values in the interval $u=1, \ldots, W$. Similarly to the size of bursts $\Delta$, their duration $W$ is also a stochastic quantity which varies over a broad range. It can be observed in Fig. 6 that the probability distribution $p(W)$ of the burst duration $W$ has the same functional form as $p(\Delta)$, i.e., power-law behavior followed by an exponential cutoff is evidenced. In higher dimensions the system can tolerate larger bursts of longer duration, hence, the power-law exponent $\tau_{W}$ of $p(W)$ decreases from $\tau_{W}=5.7$ of $D=1$ to the vicinity of $\tau_{W} \approx 4$ for $D=8$, while the cutoff duration gradually increases with the dimensionality.

For single bursts $\Delta_{S}(u)$ is a stochastic curve, hence, quantitative characterization of the internal dynamics of avalanches is provided by the average temporal profile $\left\langle\Delta_{s}(u, W)\right\rangle$, where the step size $\Delta_{s}$ is averaged at fixed values of $u$ for avalanches of the same duration W [17]. Average profiles are illustrated in

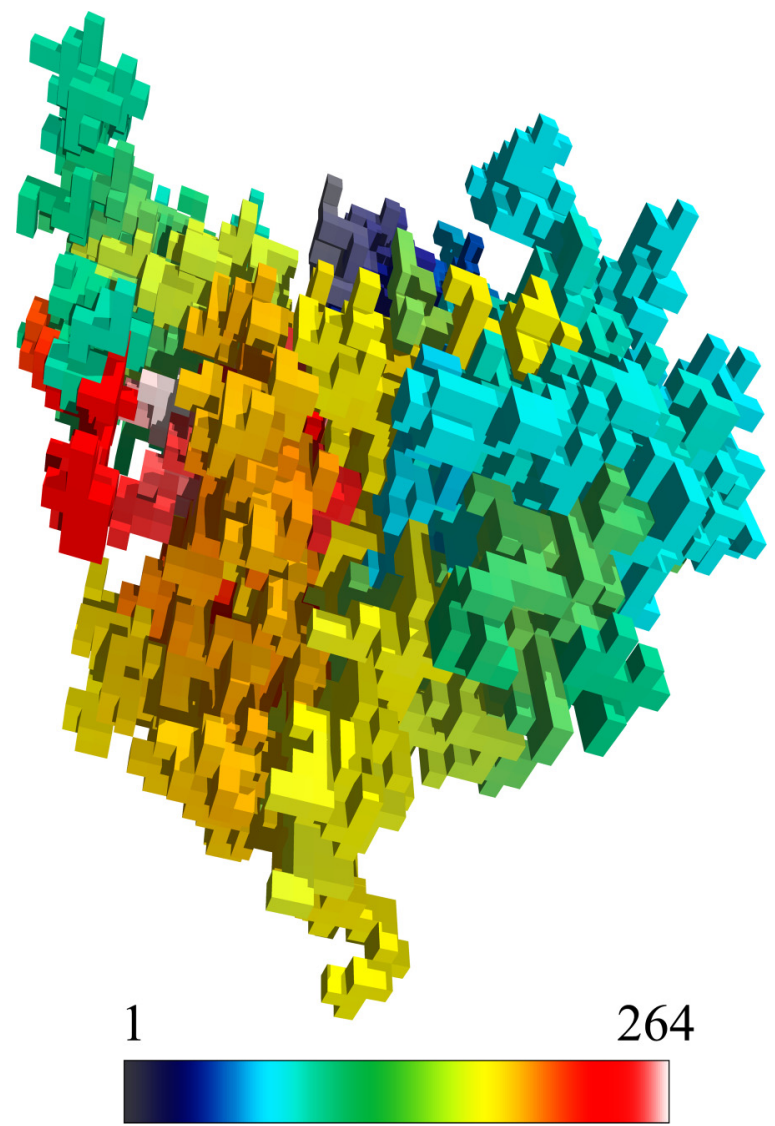

FIG. 5. The temporal evolution of a single burst of size $\Delta=$ 8705 and duration $W=264$ in a three-dimensional bundle. Single fibers are represented by cubes, which are colored according to the growth steps they belong to.

Fig. 7 for different durations $W$ for all dimensions considered. It can be observed that, except for $D=1$, the shape of avalanches obtained is similar to the experimental findings $[6,7]$. For $D=1$ the stress concentration is so high at the tip of

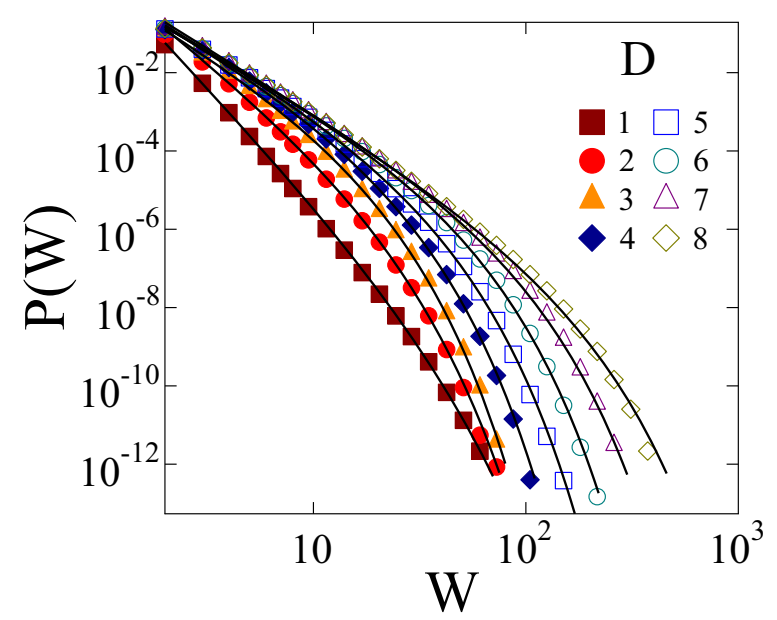

FIG. 6. Probability distribution $p(W)$ of the duration $W$ of avalanches for different dimensions $D$. The continuous lines represent fits with the functional form of Eq. (6). 

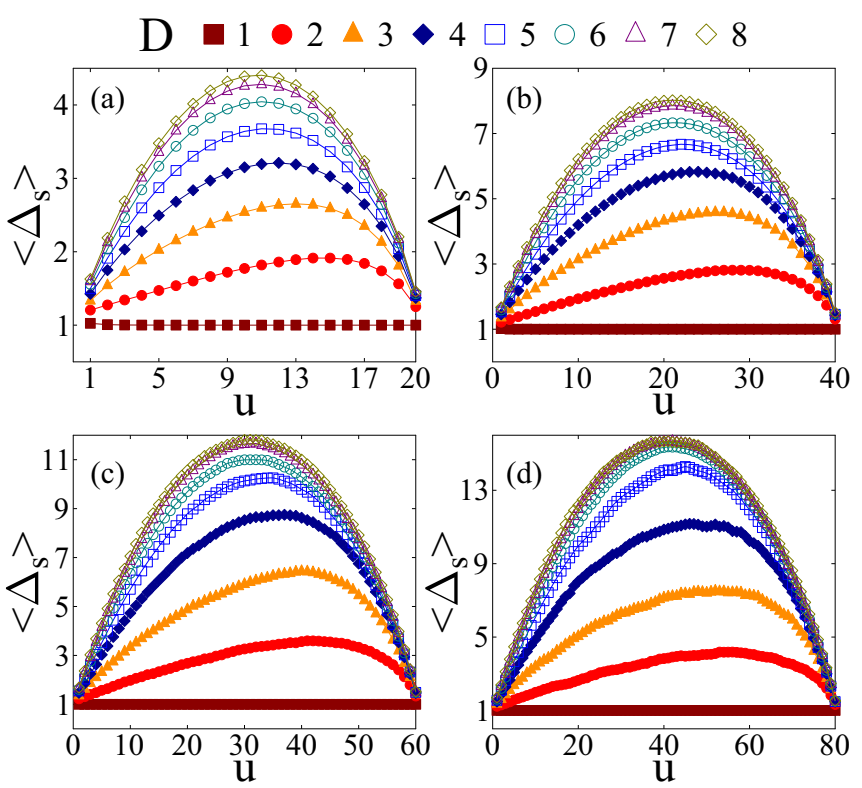

FIG. 7. Average temporal profile of avalanches of different durations for all the dimensions $D$ considered ( $D$ increases from bottom to top) for four different durations $W: 20$ (a), 40 (b), 60 (c), 80 (d). For the special case of $D=1$ the flat profile means that each load redistribution step causes the breaking of a single fiber. For a fixed duration, in low-dimensional space the profiles have a strong right-handed asymmetry which gradually disappears with increasing $D$.

growing broken clusters that all steps of the breaking sequence have a size $\Delta_{s}=1$, since a larger number of breaking fibers would trigger a catastrophic avalanche. Consequently, the emerging pulse profile is completely flat. At low dimensions $D=2,3$ the profiles have a strong right-handed asymmetry at all durations $W$, which means that these bursts start slowly, and they gradually accelerate, while their stopping is more sudden. As the dimensionality of the system increases, the degree of asymmetry decreases, and eventually a symmetric parabolic shape is obtained which is characteristic for mean field avalanches $[1,8,17]$. Recently, similar asymmetric avalanche shapes have been obtained in a fiber bundle model of creep rupture with localized load sharing [17]. Since in those calculations the external load was fixed, bursts were triggered by aging-induced slow breaking of fibers. However, the cascading breaking sequence of avalanches had essentially the same dynamics as in the present study. It has been shown in Ref. [17] for $D=2$ that the short-range load sharing and the heterogeneous stress field built up along the perimeter of cracks are responsible for the right-handed asymmetry of pulse profiles.

Comparing the curves in Fig. 7 for a fixed dimension it can be inferred that bursts of a longer duration $W$ have a larger average height $\left\langle\Delta_{s}^{\max }\right\rangle$ and average size $\langle\Delta\rangle$. Figure 8 demonstrates that rescaling the pulse profiles with an appropriate power of $W$ pulses of different duration can be collapsed on the top of each other. The good quality data collapse implies the scaling form

$$
\left\langle\Delta_{s}(u, W)\right\rangle=W^{\alpha} f(u / W),
$$
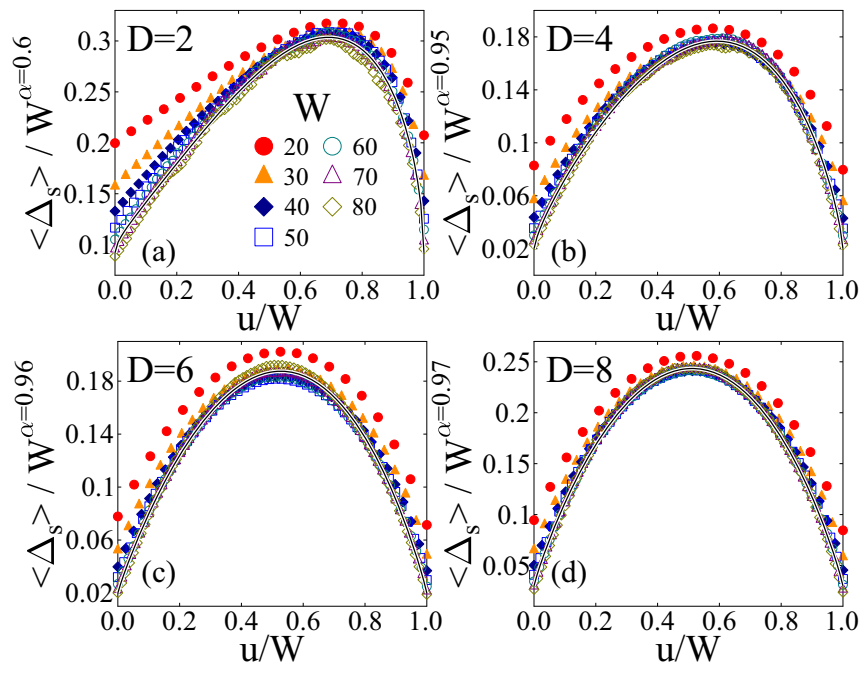

FIG. 8. Rescaling the pulse profiles for a fixed dimension with an appropriate power of $W$ profiles of different durations can be collapsed. Except for the shortest duration $W=20$ good quality collapse is obtained which improves with increasing dimension $D$. The continuous lines represent fits of the scaling function with Eq. (11).

where the value of the exponent $\alpha$ and the scaling function $f(x)$ both depend on the dimensionality of the system $D$. In Fig. 8 the value of $\alpha$ was tuned to achieve the best collapse. Deviations from the scaling function $f(x)$ occur for the shortest durations, which confirms that Eq. (8) is asymptotically valid.

It follows from the scaling structure Eq. (8) that the average avalanche size has a power-law dependence on the duration $[1,12,17,35,36]$

$$
\langle\Delta\rangle \sim W^{1+\alpha},
$$

which provides an alternative way to determine the exponent $\alpha$, as well. Figure 9 shows that the asymptotic behavior of the average size of bursts $\langle\Delta\rangle$ of the same duration $W$ can be well described by a power law. The value of the exponent $1+\alpha$ obtained by fitting the $\langle\Delta\rangle(W)$ curves with Eq. (9) is presented in Fig. 10 as a function of the embedding dimension $D$. Since in $D=1$ the step size $\Delta_{s}$ does not exceed 1 , the total size $\Delta$ of an avalanche is proportional to its duration so that $1+\alpha=1$ and $\alpha=0$ follows. As $D$ increases, $1+\alpha$ gradually approaches 2 , and hence $\alpha$ tends to its mean field value $\alpha=1$. Based on Eq. (9) a scaling relation can be established between $\alpha$ and the exponents $\tau$ and $\tau_{W}$ of the probability distribution of the size and duration of bursts

$$
1+\alpha=\left(\tau_{W}-1\right) /(\tau-1) .
$$

In Fig. 10 we compare the prediction of the scaling law by substituting the numerical values of $\tau$ and $\tau_{W}$ on the righthand side of Eq. (10) by the value of $1+\alpha$ obtained by direct fitting of the average burst size in Fig. 9. A reasonable agreement can be observed between the two curves, which confirms the consistency of the results.

Avalanche profiles $\left\langle\Delta_{s}(u, W)\right\rangle$ have an asymmetric functional form in all dimensions; however, the degree of asymmetry depends on the embedding dimension $D$. In Ref. [6] the 


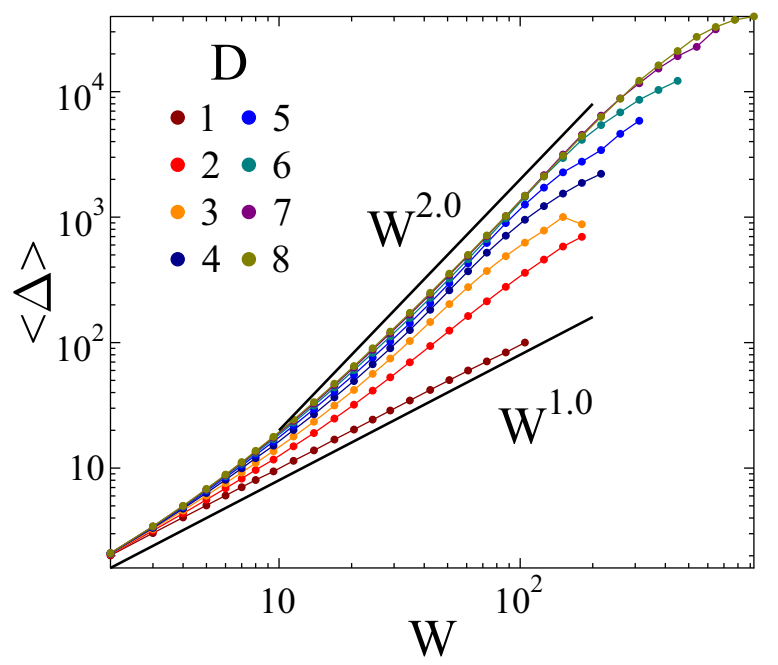

FIG. 9. Average size of bursts as a function of their duration for different embedding dimensions $D$. The value of the power-law exponent increases from one to two as the dimensionality increases from one. The value of $D$ monotonically increases from the bottom curve to the top one.

following expression has recently been suggested to quantify avalanche shapes:

$$
f(x) \sim[x(1-x)]^{\alpha}\left[1-a\left(x-\frac{1}{2}\right)\right] .
$$

Note that the scaling laws (8) and (9) are consistent with the generic form of Eq. (11) with the same value of the exponent $\alpha$. The pulse asymmetry is represented by the parameter $a$ such that zero value of $a$ implies symmetry, while negative and positive values of $a$ capture right- and left-handed asymmetry, respectively. It can be observed in Fig. 8 that Eq. (11) provides excellent fits of pulse profiles for all dimensions. Figures 11 and 12 present the value of the parameters $\alpha$ and $a$ obtained

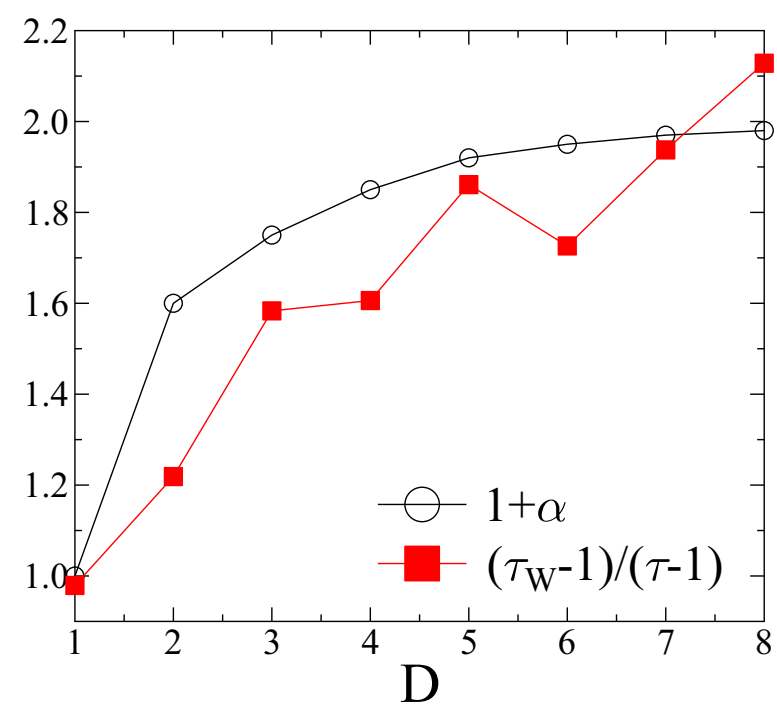

FIG. 10. The value of the exponent $1+\alpha$ of Eq. (9) compared to the outcome of the scaling relation Eq. (10). The good agreement of the two curves confirms the consistency of the results.

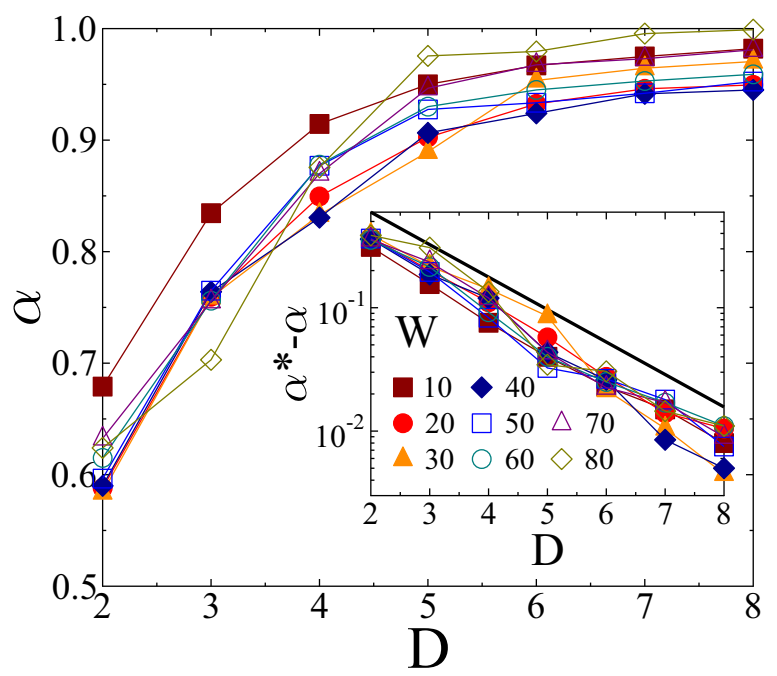

FIG. 11. The $\alpha$ parameter of the avalanche profile described by Eq. (11) obtained for avalanches of different durations $W$ as function of the embedding dimension $D$. The inset shows that subtracting $\alpha$ from a limit value $\alpha^{*}$ a straight line is obtained on a semilogarithmic plot, which implies an exponential dependence on the dimensionality. The straight line represents the exponential with the parameter $D^{*}=1.65$.

by fitting for several avalanche durations $W$. The careful analysis revealed that the parameter values practically do not depend on the avalanche duration $W$, except for some statistical fluctuations the numerical values of $\alpha$ and $a$ agree with each other at different $W$. In agreement with the results presented in Fig. 10, for low dimensions the exponent $\alpha$ starts from the vicinity of $0.6-0.7$, and it increases to $0.95-1$ at high dimensions (see Fig. 11). At the same time the observed right-handed asymmetry of profiles in Fig. 7 gives rise to

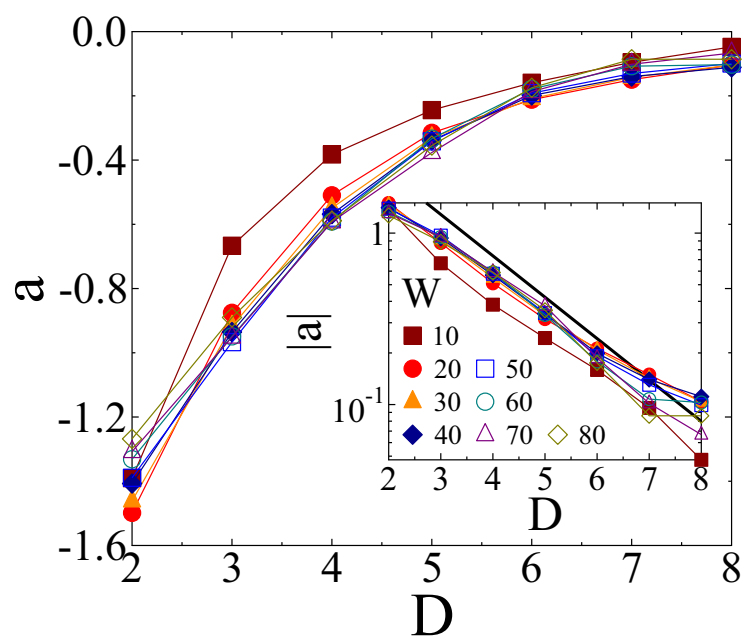

FIG. 12. The asymmetry parameter $a$ of the avalanche profile of Eq. (11) obtained for avalanches of different durations $W$ as function of the embedding dimension $D$. The inset presents the absolute value of $a$ on a semilogarithmic plot. The straight line implies that $a$ approaches zero with an exponential dependence on $D$. The straight line represents the exponential with the parameter $D^{*}=1.65$. 
negative values of $a$ in Fig. 12. As the event duration $W$ increases from 10 to 80, in Figs. 11 and 12 the numerical error of the determination of the parameters $\alpha$ and $a$ increases from 0.05 to 0.15 , and from 0.07 to 0.16 , respectively. The reason is that due to the rapidly decreasing duration distribution $p(W)$ of bursts, the statistics of events of higher duration decreases. In higher dimensions $\alpha$ approaches one and $a$ increases to the vicinity of zero, which imply that the profile shape evolves towards a simple symmetric parabola of mean field crackling systems $[8,12,17,36]$. The inset of Fig. 11 demonstrates that subtracting $\alpha$ from a proper limit value $\alpha^{*}$ straight lines are obtained on a semilogarithmic plot. For each $W$ the value of $\alpha^{*}$ was varied in the range $0.96-1.01$ independently until best straight lines were achieved. Similarly, the inset of Fig. 12 presents the absolute value of $a$ where again an exponential dependence is evidenced. The results imply that the behavior of the pulse parameters $\alpha$ and $a$ is consistent with that of the macroscopic strength and avalanche exponent, i.e., they approach their mean field values with an exponential dependence on the dimensionality.

\section{DISCUSSION}

The fracture of heterogeneous materials is strongly affected by the degree of disorder, which controls the spatial variation of microscopic materials' strength, and the stress fluctuations emerging due to localized stress redistribution after microfracturing events. The competition of these two sources of disorders, i.e.. strength and stress disorders, gives rise to a highly complex fracture process which manifests itself in the variation of macroscopic strength, in the statistics of crackling bursts, and in the temporal evolution of single crackling events. At a fixed amount of quenched strength disorder stress fluctuations are mediated by the range of load redistribution. Former studies have revealed two universality classes of fracture, i.e., the localized load-sharing (LLS) class and the mean field class (ELS), characterized by a high degree of brittleness and a quasibrittle response with a large amount of avalanche precursors of failure, respectively. In order to understand the competing role of different disorder sources between the limiting cases of the LLS and ELS classes, in our study we considered a fiber bundle model with a fixed amount of strength disorder and varied the dimensionality of the system from one to eight at a fixed range of load sharing. The strength disorder is represented by an exponential distribution of the failure threshold of fibers, which falls in the well-understood universality classes in both the ELS and LLS limits of load sharing. In all dimensions nearest neighbor load redistribution was implemented on cubic lattices with periodic boundary conditions in all lattice directions.

Our study revealed a very interesting dimensional crossover between the two universality classes of fracture: Both on the macro- and microlevels fracture characteristics evolve with the dimensionality from the highly brittle response of low-dimensional systems controlled by stress fluctuations, to the quasibrittle behavior in high dimensions where the strength disorder dominates. For the macroscopic strength of the bundle and for the power-law exponent of the size distribution of crackling bursts the convergence to the mean field limit is described by an exponential functional form.
Avalanche profiles have been found before to be very sensitive to models' details, especially to the degree of correlations of microscopic events leading to collective avalanches of local failures. We showed that the symmetry of avalanche profiles depends on the dimensionality of the system, gradually shifting from a strongly asymmetric shape at low dimensions to a symmetric parabolic form in the mean field limit. The parameters of pulse profiles were found to evolve towards their mean field values with an exponential dependence on the dimensionality similarly to the macroscopic strength and avalanche exponent of the system.

Increasing the dimensionality of the bundle implies a decreasing stress concentration along broken clusters by increasing the connectivity of the system. As a consequence, stress fluctuations have a diminishing role with increasing dimension giving more room for the quenched disorder of fibers' strength. The evolution of avalanche temporal profiles we observe with the embedding dimension is similar to what has been obtained recently for avalanches of a propagating crack front when increasing the range of interaction in a fracture model of fixed $D=2$ dimensions [6].

Recently, the effect of the long-range connection of fibers on the fracture process has been studied. Instead of a regular lattice, fibers were assigned to the nodes of a complex network with small world properties [37]. Redistributing the load along the edges of the network simulations revealed that a small amount of long-range connection is sufficient to converge the system from the LLS to the mean field universality class. Eventually, the dominance of quenched structural disorder is responsible for the ELS behavior similarly to our case.

Fiber bundles in higher dimensions have recently been investigated in Ref. [18]. Focusing on the amount of damage accumulated up to failure and on the distribution of avalanches a crossover from the LLS to the mean field universality class was pointed out. However, the crossover is described by a power-law functional form contrary to our exponential behavior. This difference may arise due to the different protocols of load redistribution used in the simulations. In agreement with Ref. [18], our results imply that the upper critical dimension of the fracture of heterogeneous materials is infinite. Further support of this remarkable feature of fracture phenomena could be obtained by a finite size scaling analysis in higher dimensions; however, it turned to be infeasible due to the overwhelming numerical costs.

In the broader context of critical phenomena, the absence of a finite upper critical dimension has also been found in Kardar-Parisi-Zhang type surface growth models [38], where the critical exponents present an approximate exponential dependence on the dimensionality of the system [39]. We conjecture that the quenched disorder of the system and the locally conserving nature of the dynamics (i.e., the entire load dropped by broken fibers is redistributed over the intact ones without loss) are responsible for the absence of a finite upper critical dimension beyond which mean field behavior is attained. The exponential crossover from the local to the mean field universality class of fracture involves a characteristic dimension $D^{*}$ which falls between one and two. Since avalanche shapes of the one-dimensional system do not conform with the higher dimensional ones, we propose the 
interpretation of $D^{*}$ as the lower critical dimension of fracture phenomena. Our simulations confirm that for $D>D^{*}$ all characteristic quantities of the system evolve through gradual quantitative changes, but the qualitative behavior remains robust as the embedding dimension increases.

\section{ACKNOWLEDGMENTS}

The work is supported by the EFOP-3.6.1-16-2016-00022 project. The project is cofinanced by the European Union and the European Social Fund. This research was supported by the National Research, Development and Innovation Fund of Hungary, financed under the K-16 funding scheme Project No. K 119967 and K 109577. This work was supported through the New National Excellence Program of the Ministry of Human Capacities of Hungary. The research was financed by the Higher Education Institutional Excellence Programme of the Ministry of Human Capacities in Hungary, within the framework of the Energetics thematic program of the University of Debrecen.
[1] J. P. Sethna, K. A. Dahmen, and C. R. Meyers, Nature (London) 410, 242 (2001).

[2] M. Alava, P. K. Nukala, and S. Zapperi, Adv. Phys. 55, 349 (2006).

[3] S. Santucci, R. Planet, K. J. Maloy, and J. Ortin, EPL (Europhys. Lett.) 94, 46005 (2011).

[4] P. D. Ispánovity, L. Laurson, M. Zaiser, I. Groma, S. Zapperi, and M. J. Alava, Phys. Rev. Lett. 112, 235501 (2014).

[5] G. Durin and S. Zapperi, Phys. Rev. Lett. 84, 4705 (2000).

[6] L. Laurson, X. Illa, S. Santucci, K. Tore Tallakstad, K. J. Maloy, and M. J. Alava, Nat. Commun. 4, 4927 (2013).

[7] Z. Danku, G. B. Lenkey, and F. Kun, Appl. Phys. Lett. 106, 064102 (2015).

[8] K. A. Dahmen, Y. Ben-Zion, and J. T. Uhl, Nat. Phys. 7, 554 (2011).

[9] M. Miguel, A. Vespignani, S. Zapperi, J. Weiss, and J. Grasso, Nature (London) 410, 667 (2001).

[10] F. Colaiori, Adv. Phys. 57, 287 (2008).

[11] N. Friedman, S. Ito, B. A. W. Brinkman, M. Shimono, R. E. L. DeVille, K. A. Dahmen, J. M. Beggs, and T. C. Butler, Phys. Rev. Lett. 108, 208102 (2012).

[12] S. Zapperi, C. Castellano, F. Colaiori, and G. Durin, Nature Phys. 1, 46 (2005).

[13] S. Papanikolaou, F. Bohn, R. L. Sommer, G. Durin, S. Zapperi, and J. P. Sethna, Nat. Phys. 7, 316 (2011).

[14] L. Laurson, S. Santucci, and S. Zapperi, Phys. Rev. E 81, 046116 (2010).

[15] K. J. Måløy, S. Santucci, J. Schmittbuhl, and R. Toussaint, Phys. Rev. Lett. 96, 045501 (2006).

[16] F. Kun, G. B. Lenkey, N. Takács, and D. L. Beke, Phys. Rev. Lett. 93, 227204 (2004).

[17] Z. Danku and F. Kun, Phys. Rev. Lett. 111, 084302 (2013).

[18] S. Sinha, J. T. Kjellstadli, and A. Hansen, Phys. Rev. E 92, 020401 (2015).

[19] S. Pradhan, A. Hansen, and B. K. Chakrabarti, Rev. Mod. Phys. 82, 499 (2010).

[20] A. Hansen, P. Hemmer, and S. Pradhan, The Fiber Bundle Model: Modeling Failure in Materials, Statistical Physics of Fracture and Breakdown (Wiley, New York, 2015).
[21] F. Kun, F. Raischel, R. C. Hidalgo, and H. J. Herrmann, in Modelling Critical and Catastrophic Phenomena in Geoscience: A Statistical Physics Approach, Lecture Notes in Physics, edited by P. Bhattacharyya and B. K. Chakrabarti (Springer-Verlag, Berlin, 2006), pp. 57-92.

[22] M. Kloster, A. Hansen, and P. C. Hemmer, Phys. Rev. E 56, 2615 (1997).

[23] R. C. Hidalgo, F. Kun, K. Kovács, and I. Pagonabarraga, Phys. Rev. E 80, 051108 (2009).

[24] R. C. Hidalgo, Y. Moreno, F. Kun, and H. J. Herrmann, Phys. Rev. E 65, 046148 (2002).

[25] F. Raischel, F. Kun, and H. J. Herrmann, Phys. Rev. E 74, 035104 (2006).

[26] K. Kovács, R. C. Hidalgo, I. Pagonabarraga, and F. Kun, Phys. Rev. E 87, 042816 (2013).

[27] R. L. Smith, Proc. R. Soc. Lond. A 372, 539 (1980).

[28] L. N. McCartney and R. L. Smith, J. Appl. Mech 50, 601 (1983).

[29] V. Kádár, Z. Danku, and F. Kun, Phys. Rev. E 96, 033001 (2017).

[30] A. Garcimartin, A. Guarino, L. Bellon, and S. Ciliberto, Phys. Rev. Lett. 79, 3202 (1997).

[31] M. Stojanova, S. Santucci, L. Vanel, and O. Ramos, Phys. Rev. Lett. 112, 115502 (2014).

[32] D. Bonamy, S. Santucci, and L. Ponson, Phys. Rev. Lett. 101, 045501 (2008).

[33] K. T. Tallakstad, R. Toussaint, S. Santucci, J. Schmittbuhl, and K. J. Måløy, Phys. Rev. E 83, 046108 (2011).

[34] S. L. Phoenix and W. I. Newman, Phys. Rev. E 80, 066115 (2009).

[35] A. P. Mehta, A. C. Mills, K. A. Dahmen, and J. P. Sethna, Phys. Rev. E 65, 046139 (2002).

[36] A. Baldassarri, F. Colaiori, and C. Castellano, Phys. Rev. Lett. 90, 060601 (2003).

[37] D.-H. Kim, B. J. Kim, and H. Jeong, Phys. Rev. Lett. 94, 025501 (2005).

[38] G. Ódor, Universality in Nonequilibrium Lattice Systems: Theoretical Foundations (World Scientific, Singapore, 2008).

[39] G. Ódor, B. Liedke, and K.-H. Heinig, Phys. Rev. E 81, 031112 (2010). 